\title{
VLBI for Gravity Probe B. IV. A New Astrometric Analysis Technique and a Comparison with Results from Other Techniques
}

\section{Citation}

Lebach, D. E., N. Bartel, M. F. Bietenholz, R. M. Campbell, D. Gordon, J. I. Lederman, J.-F. Lestrade, R. R. Ransom, M. I. Ratner, and I. I. Shapiro. 2012. "VLBI for Gravity Probe B. IV. A New Astrometric Analysis Technique and a Comparison with Results from Other Techniques." The Astrophysical Journal Supplement Series 201, no. 1: 1-13.

\section{Published Version}

doi:10.1088/0067-0049/201/1/4

\section{Permanent link}

http://nrs.harvard.edu/urn-3:HUL.InstRepos:11858833

\section{Terms of Use}

This article was downloaded from Harvard University's DASH repository, and is made available under the terms and conditions applicable to Other Posted Material, as set forth at http:// nrs.harvard.edu/urn-3:HUL.InstRepos:dash.current.terms-of-use\#LAA

\section{Share Your Story}

The Harvard community has made this article openly available.

Please share how this access benefits you. Submit a story.

Accessibility 


\title{
VLBI FOR GRAVITY PROBE B. IV. A NEW ASTROMETRIC ANALYSIS TECHNIQUE AND A COMPARISON WITH RESULTS FROM OTHER TECHNIQUES
}

\author{
D. E. Lebach ${ }^{1}$, N. Bartel ${ }^{2}$, M. F. Bietenholz ${ }^{2,6}$, R. M. CAmpbell ${ }^{3}$, D. Gordon ${ }^{4}$, J. I. Lederman ${ }^{2}$, \\ J.-F. Lestrade ${ }^{5}$, R. R. RANSOM ${ }^{2,7}$, M. I. RATNER ${ }^{1}$, AND I. I. SHAPIRO ${ }^{1}$ \\ ${ }^{1}$ Harvard-Smithsonian Center for Astrophysics, 60 Garden Street, Cambridge, MA 02138, USA \\ ${ }^{2}$ Department of Physics and Astronomy, York University, 4700 Keele Street, Toronto, ON M3J 1P3, Canada \\ ${ }^{3}$ Joint Institute for VLBI in Europe, Oude Hoogeveensedijk 4, 7991 PD Dwingeloo, The Netherlands \\ ${ }^{4}$ NVI Inc./NASA Goddard Space Flight Center, Greenbelt, MD 20771, USA \\ ${ }^{5}$ Observatoire de Paris, Centre National de la Recherche Scientifique, 77 Av. Denfert Rochereau, 75014 Paris, France \\ Received 2011 May 31; accepted 2011 June 13; published 2012 June 7
}

\begin{abstract}
When very long baseline interferometry (VLBI) observations are used to determine the position or motion of a radio source relative to reference sources nearby on the sky, the astrometric information is usually obtained via (1) phase-referenced maps or (2) parametric model fits to measured fringe phases or multiband delays. In this paper, we describe a "merged" analysis technique which combines some of the most important advantages of these other two approaches. In particular, our merged technique combines the superior model-correction capabilities of parametric model fits with the ability of phase-referenced maps to yield astrometric measurements of sources that are too weak to be used in parametric model fits. We compare the results from this merged technique with the results from phase-referenced maps and from parametric model fits in the analysis of astrometric VLBI observations of the radio-bright star IM Pegasi (HR 8703) and the radio source B2252+172 nearby on the sky. In these studies we use central-core components of radio sources 3C 454.3 and B2250+194 as our positional references. We obtain astrometric results for IM Peg with our merged technique even when the source is too weak to be used in parametric model fits, and we find that our merged technique yields astrometric results superior to the phase-referenced mapping technique. We used our merged technique to estimate the proper motion and other astrometric parameters of IM Peg in support of the NASA/Stanford Gravity Probe B mission.
\end{abstract}

Key words: astrometry - binaries: close - radio continuum: stars - stars: activity - stars: imaging - stars: individual (IM Pegasi) - techniques: interferometric

\section{INTRODUCTION}

Very long baseline interferometry (VLBI) provides the most accurate astrometric measurements of celestial objects currently attainable. VLBI astrometry has been used, among other applications, to define the most nearly inertial reference frame available for the positions of celestial objects (e.g., Ma et al. 1998; Fey et al. 2009), tie an inertial (extragalactic) reference frame to a planetary ephemeris via observations of pulsars (e.g., Bartel et al. 1985; Rodin \& Sekido 2002; Dodson et al. 2003), characterize motions and other properties of the Earth (e.g., Ryan et al. 1993; Mathews \& Shapiro 1995), study positions and motions of maser spots in galaxies as a means of estimating black hole masses (e.g., Ishihara et al. 2001; Kondratko et al. 2004) and distances to other galaxies (Herrnstein et al. 1999), and test general relativity via measurements of solar gravitational deflection (e.g., Counselman et al. 1974; Shapiro et al. 2004). In this paper, we describe the analysis of astrometric VLBI observations of the RS CVn binary star IM Pegasi (IM Peg; HR 8703), the radio-bright star which served as the "guide star," and hence as the positional reference, for the NASA/Stanford Gravity Probe B $(G P-B)$ experiment.

$G P-B$ was designed to measure the geodetic and framedragging effects predicted by general relativity (see Paper I, Shapiro et al. 2012), as manifested in secular changes in

\footnotetext{
6 Now also at Hartebeesthoek Radio Astronomy Observatory, PO Box 443, Krugersdorp 1740, South Africa.

7 Now at Okanagan College, 583 Duncan Avenue West, Penticton, BC V2A 2K8, Canada and also at the National Research Council of Canada, Herzberg Institute of Astrophysics, Dominion Radio Astrophysical Observatory, PO Box 248, Penticton, BC V2A 6K3, Canada.
}

the spin-axis orientations of four gyroscopes placed within a spacecraft in a low-altitude, polar orbit about the Earth. The spacecraft also had an onboard telescope equipped with a tracking system designed to keep the guide star, IM Peg, at the center of the telescope field of view, and thereby provide a directional reference: the relativistic effects were estimated by measuring the drift rates in the spin-axis directions of the four gyroscopes relative to the direction to IM Peg. Thus, if any motions of IM Peg on the sky relative to an inertial frame were not accounted for sufficiently accurately, they could map directly into the gyro drift-rate signals and thereby corrupt the relativistic measurements. (Ideally the $G P-B$ guide star would have been a distant quasar, which could be treated as effectively motionless on the sky, rather than a star in our galaxy, but the onboard telescope could only track objects brighter than about sixth magnitude, so a relatively bright star was the only possible choice. A list of criteria that had to be met by the guide star and a description of the selection process that led to the choice of IM Peg is in Paper I.) Our VLBI observations of IM Peg were therefore undertaken to determine the motions of the star relative to an inertial frame so that the relativistic effects included in the gyro measurements could be properly separated. The goal of our VLBI observations was to determine, relative to an inertial frame, the proper motion of IM Peg with a standard error of 0.14 mas $\mathrm{yr}^{-1}$ or less in each of the north-south and east-west directions. This accuracy goal was based upon an error projection for the full $G P-B$ experiment prior to launch in 2004 April and the desire at the time for the uncertainty in the proper motion of IM Peg not to constitute a significant source of error for the experiment. 
In this paper, we present a new approach for astrometric VLBI data analysis that we developed to meet the accuracy goal for $G P-B$. We then compare the results from this approach with the results from two well-established VLBI analysis techniques: phase-referenced mapping (PRM) and "phase connection" followed by parametric model fitting (PMF). Our new approach is a combination of these two standard approaches that provides many of the benefits of each. All three approaches use differential VLBI astrometry, in which the target source (i.e., the source of astrometric interest) is observed alternately with at least one extragalactic, compact reference source nearby on the sky to provide model corrections in the analysis of the target-source data. The way these corrections are applied differs among the approaches, but in all cases the astrometric measurements of the target source are relative to the position(s) of the reference source(s).

In Section 2, we briefly describe the VLBI observables used in our analysis. We then discuss some basic aspects of the PRM and PMF analysis techniques in Sections 3 and 4, respectively. In Section 5, we describe our new "merged" analysis technique and then our implementation of it. We compare the results from the three techniques in Section 6, and offer corresponding conclusions in Section 7.

\section{VLBI OBSERVABLES: FRINGE PHASE, FRINGE RATE, AND MULTIBAND DELAY}

In VLBI observations of a compact source, the total fringe phase (or visibility phase, or interferometric phase, or simply "phase"), $\phi(\omega, t)$, is the phase at frequency $\omega$ and time $t$ that is associated with the difference in arrival times of signals received from the source at two antennas in a VLBI array. We describe $\phi(\omega, t)$ as

$$
\begin{aligned}
\phi(\omega, t)=\omega\left[\tau_{\text {geom }}(t)+\tau_{\text {inst }}(\omega, t)+\tau_{\text {atm }}(\omega, t)\right. \\
\left.+\tau_{\text {struc }}(\omega, t)+\tau_{\text {noise }}(\omega, t)\right]+2 \pi N(\omega, t),
\end{aligned}
$$

where $\tau_{\text {geom }}(t)$, the "geometric delay," is the difference in the signal arrival times in vacuum at the two antennas; $\tau_{\text {inst }}(\omega, t)$ represents the difference in the instrumental delays (including clock behavior) at the two antenna sites; $\tau_{\mathrm{atm}}(\omega, t)$ represents the difference in signal propagation times to the two antennas due to all atmospheric effects, including the contributions of the hydrostatic (or "dry") atmospheric constituents, atmospheric water vapor, and the ionosphere; $\tau_{\text {struc }}(\omega, t)$ is the delay contribution from source structure, i.e., from the nonpointlike brightness distribution of the source; $\tau_{\text {noise }}(\omega, t)$ represents the (thermal) noise contribution to the phase measurement; and $N(\omega, t)$ represents the integer number of "ambiguities," or "phase wraps," included in the measurement. Information about the number of phase wraps associated with a fringe phase is not inherent in a measurement of fringe phase, and in general a measured fringe phase can be defined such that $-\pi<\phi_{\text {meas }}(\omega, t) \leqslant \pi$ or $0 \leqslant \phi_{\text {meas }}(\omega, t)<2 \pi$. There is a fringe phase associated with each antenna pair, or "baseline," within the VLBI array. The "fringe rate" is the partial derivative of the fringe phase with respect to time. The "multiband delay" is a measured approximation of the group delay, which is the partial derivative of the fringe phase with respect to frequency.

To lowest order (i.e., neglecting, e.g., relativistic effects), the geometric delay is given by

$$
\tau_{\mathrm{geom}}(t)=\frac{1}{c}[\mathbf{B}(t) \cdot \hat{\mathbf{s}}(t)],
$$

where $c$ is the speed of light in vacuum, $\mathbf{B}(t)$ is the threedimensional vector between the two antennas of the baseline, and $\hat{\mathbf{s}}(t)$ is the unit vector in the direction of the observed source. All of the astrometric information in the measured fringe phase is contained in $\tau_{\text {geom }}(t)$. (While we do not show the relativistic contributions to $\tau_{\text {geom }}(t)$ in Equation (2), we do include those contributions throughout our analyses. We also account for Earth motions relative to the solar system barycenter during the time between signal arrival at the two antennas.)

The model we used for $\tau_{\mathrm{atm}}(\omega, t)$ can be more explicitly described by

$$
\begin{aligned}
\tau_{\mathrm{atm}_{\mathrm{A}}}(\omega, t, \epsilon, \hat{\mathbf{s}})= & \tau_{\mathrm{zen}_{\text {_dry }}}(t) m_{\mathrm{dry}_{\mathrm{A}}}(t, \epsilon)+\tau_{\mathrm{zen}_{-} \mathrm{wet}_{\mathrm{A}}}(t) m_{\mathrm{wet}_{\mathrm{A}}}(t, \epsilon) \\
& +\tau_{\text {ion }_{\mathrm{A}}}(\omega, t, \hat{\mathbf{s}}),
\end{aligned}
$$

where the " $A$ " subscript refers to site A; $\tau_{\text {zen_dry }_{A}}(t)$ is the propagation delay through the atmosphere at zenith under the assumption that the atmosphere is in hydrostatic equilibrium; $\tau_{\text {zen_wet }_{\mathrm{A}}}(t)$ is the additional propagation delay through the atmosphere at zenith due to tropospheric water vapor (Davis et al. 1985); $m_{\mathrm{dry}_{\mathrm{A}}}(t, \epsilon)$ and $m_{\mathrm{wet}_{\mathrm{A}}}(t, \epsilon)$ are, respectively, the "mapping functions" that project (i.e., scale) the "dry" and "wet" delays at zenith to the line-of-site elevation, $\epsilon$, of the observed source; and $\tau_{\text {ion }_{\mathrm{A}}}(\omega, t, \hat{\mathbf{s}})$ denotes the line-of-site contribution of the ionosphere to phase delays in the direction of the observed source.

\section{PHASE-REFERENCED MAPPING (PRM)}

The basic idea behind the PRM technique is to use the data from the observed reference sources to account for otherwise unmodeled fluctuations in instrumental or atmospheric delays, as well as for other model errors. Specifically, the "residual" fringe phases and rates, i.e., the differences between the measured and a priori model values of these quantities, are obtained for the reference sources and then temporally and sometimes also spatially interpolated to the observation time and sky position of the target source to estimate the effects of model errors on the target-source observables (see, e.g., Shapiro et al. 1979; Gorenstein et al. 1983; Lestrade et al. 1990; Beasley \& Conway 1995; Fomalont 2005). Typically the cycle time over which the reference sources and target source are observed is relatively short, from several seconds to several minutes. To the extent that reference-source structure is properly accounted for and the reference-source residuals are properly interpolated to the observation time and sky position of the target source, the remaining residual components in the target-source data can be attributed primarily to measurement noise, unmodeled targetsource structure, and a position offset of the target source relative to the a priori model position. Perhaps the most important feature of the PRM technique is that the target-source data can be coherently integrated over the entire span of an observing session. Thus, even very weak sources with flux densities well under $1 \mathrm{mJy}$ can be detected and imaged with VLBI via this technique.

Henceforth in this paper, a "scan" refers to a single continuous observation of a particular source. Fringe-phase and fringerate measurements from successive reference-source scans can provide proper model fringe-phase adjustments for intervening target-source data only if the change in the model errors between the scans is $\ll 2 \pi$, so that the number of $2 \pi$ phase wraps between successive reference-source scans can be accurately tracked. For that reason, the cycle time over which the reference and target sources are observed should be as short as possible. On the 
other hand, the reference-source scans must be long enough to provide reliable fringe-phase measurements from single scans, and the scan times for the target source should be long enough to ensure sufficient coherent integration time over the course of the observing session to produce an image with acceptably high signal-to-noise ratio $(\mathrm{S} / \mathrm{N})$. These tradeoffs must be balanced to determine the "optimum" scan and cycle times in the observation schedule.

Another beneficial feature of the PRM approach is that it is relatively quick and efficient for obtaining high-accuracy astrometry, in large part due to the software packages now readily available. In our analyses we used almost exclusively the Astronomical Image Processing System (AIPS) provided by the National Radio Astronomy Observatory (NRAO) to produce our phase-referenced maps. We followed the guidelines of Diamond (1995). Specifically, we:

1. Incorporated the instrumental phase and amplitude calibrations routinely provided in log files from individual antennas. We also unweighted faulty data based upon information in these files and from operator and correlator reports.

2. Incorporated an additional constant (over time) phase adjustment to the calibration for each antenna based upon "fringe fitting" (via AIPS task FRING) of data from a selected reference scan. ${ }^{8}$ Sometimes we used multiple reference scans to provide such phase calibration adjustments for all antennas.

3. Ran a "global fringe fit" (via AIPS task FRING) on all reference-source data while applying the phase and amplitude calibrations obtained so far.

4. Ran AIPS task IMAGR, with the fits from Step 3 as calibration, to generate "self-cal" maps of the reference sources (see Paper II, Ransom et al. 2012a). We then used these self-cal maps to refine the amplitude and phase calibration as well as the data flagging. In general we repeated Steps 3 and 4 several times.

5. Used AIPS task BPASS to further refine the phase and amplitude calibrations as a function of frequency.

6. Used AIPS task IMAGR with the final calibrations from Steps 1 through 5 to obtain a phase-referenced map of the target source.

Unfortunately, the use of the PRM technique for astrometry also poses some challenges, especially for campaigns such as ours in which the observations are made over many years. For example, one must take great care to assure that model components such as site positions, antenna axis offsets, and Earth orientation parameters (EOPs, i.e., X- and Y- pole positions, UT1-UTC, and nutation angles in longitude and obliquity; see, e.g., Seidelmann 1982, 1992) are modeled consistently and correctly throughout all observation sessions. (For example, if updated values of axis offsets for antennas are used in processing data from later observing sessions, then the new values will have to be incorporated into a re-analysis of data from earlier observing sessions to avoid possible systematic errors.) Furthermore,

\footnotetext{
8 The use of such constant phase adjustments based on data from selected scans is referred to as "manual" phase calibration (Diamond 1995). Even when measured phase calibrations were available in log files to account for instrumental effects, we found that we obtained better calibration across our observed bandwidth when we used manual phase calibrations after the application of our measured phase calibrations. This finding was based upon an assessment of the phase scatter across the spanned bandwidth of data from some of our 1997 and 1998 observations. As a result, we used manual phase calibrations for all data after we applied any available measured phase calibrations.
}

post-processing adjustments to model parameters can be quite cumbersome in some analysis packages, including AIPS, especially if the adjustments have to be made to a large number of experiments. In addition, without the use of multiple reference sources and special interpolation routines to handle the spatial (in particular, elevation-angle) dependencies of atmospheric delays, a simple temporal interpolation of reference-source fringe phases introduces model-correction errors that scale roughly with the angular separation between the reference source and the target source. Thus, the basic PRM technique commonly loses viability for an angular separation between reference and target sources larger than a few degrees. Finally, the PRM technique offers no inherently good way to assess the effects of systematic errors (such as those due to inaccurate modeling of the atmosphere). Reliable estimates of the true accuracy of the astrometric results can therefore be difficult to obtain (although see Pradel et al. 2006 for a comprehensive assessment specifically for the VLBA and EVN arrays). Our development and use of the "merged" technique described in Section 5 of this paper was motivated in part by our desire to overcome these drawbacks.

\section{PARAMETRIC MODEL FITTING (PMF)}

The PMF technique for analysis of VLBI data is, in all implementations with which we are familiar, essentially the method of "weighted-least-squares" estimation. For each reference-source and target-source scan, the total measured fringe phase and fringe rate are estimated for each baseline at an epoch near the center of the scan period. Any $2 \pi$ "phase jumps" between successive phase measurements are then resolved (or flagged as unresolvable) for each source and baseline. The overall integer number of $2 \pi$ differences between the phases from different sources is also resolved. Finally, the complete collection of "phase-connected" data is fit to a model in which corrections to a wide range of parameters-including instrumental delays, propagation delays through the atmosphere, and source positions - are simultaneously estimated.

To avoid the often onerous task of determining the change in the number of $2 \pi$ phase wraps between successive scans prior to model fitting, multiband delays (e.g., Clark et al. 1985) are commonly used in place of fringe phases as the principal observables with the PMF technique. The use of multiband delays comes at a significant cost in statistical (i.e., $\mathrm{S} / \mathrm{N}$-derived) measurement accuracy relative to the use of fringe phases, but the use of only bright radio sources as well as observations over wide spectral bandwidths can reduce this cost. The PMF approach with multiband delays is used by the geodetic VLBI community in virtually all of their data analyses, including their studies to define the International Celestial Reference Frame and track apparent motions of extragalactic sources (Ma et al. 1998; Fey et al. 2009). In general, the radio sources chosen for such studies are the brightest and most compact known.

The PMF technique has several advantages over the PRM technique. For example, since total measured fringe phases and rates (rather than residual fringe phases and rates) are processed in the analysis, one can make improvements or changes to a priori models used with multi-year data sets much more easily. Furthermore, the use of data from multiple reference sources to account for spatial (e.g., elevation-angle-dependent) as well as temporal phase variations is straightforward and readily implemented with existing software packages. In addition, because the PMF technique is a method of weighted-least-squares estimation of parameters that are treated as Gaussian random 
variables, one can readily obtain a measure of the sensitivity of one estimated parameter (such as a source declination) to the variation in another (such as the propagation delay through a site atmosphere) by evaluating the covariance matrix obtained with the parameter estimates. The effects on estimated parameters of other model changes are also straightforward to evaluate, so various sensitivity studies to assess systematic errors can be readily implemented.

Unfortunately, the PMF technique also has its limitations and problems. Unlike the PRM technique, the PMF technique can make use of only those data for which fringe phases for a baseline are reliably detected in a single scan. Thus, the technique is only well suited for relatively bright sources. Furthermore, the PMF technique offers no direct mechanism to account for source structure. One can assess fringe amplitudes and "closure" phases (Rogers et al. 1974) to identify the presence of significant structure, but the model corrections for structure have to be generated in a separate process. Finally, when accuracy requirements dictate that the PMF technique be used with fringe phases rather than multiband delays, the integral number of $2 \pi$ phase wraps between neighboring scans must be determined, which can be a very labor-intensive process and sometimes is not even possible.

\section{NEW MERGED ANALYSIS (MA) TECHNIQUE}

\subsection{Motivation}

We desired the most accurate astrometry that we could attain from our VLBI observations of IM Peg. Unfortunately, neither the PRM nor the PMF analysis technique adequately met our needs.

The PRM technique, as implemented with AIPS, relied upon insufficiently accurate models for our astrometric demands, in particular for $\tau_{\text {atm }}(\omega, t)$ and also for EOPs and other parameters. These models were difficult to reliably correct properly within AIPS. Also, the use of correct, consistent values for some model parameters, such as antenna coordinates and axis offsets, would have been somewhat burdensome to implement and ensure over the 8.5 year span of our VLBI observations (which are described further in Section 5.3.1). In addition, we wanted to make full use of observations of a second reference source to model the effects of atmospheric gradients on our measured fringe phases, and we had no satisfactory software tools with this capacity available to us with the PRM technique. ${ }^{9}$ Finally, our use of the PRM technique made difficult a robust assessment of several possible sources of systematic error in our astrometric results, including, for example, our sensitivity to errors in our a priori EOP values or in the various components of our atmospheric model.

We also could not use the PMF technique with all of our data, because during several observing sessions the radio emissions from IM Peg were too weak $(\lesssim 1 \mathrm{mJy})$ for the star to be detected in any single scan. In several other sessions, the star was

\footnotetext{
9 In our implementation of the PRM technique, we used the data from only a single reference source, $3 \mathrm{C} 454.3$, for phase calibration. Thus, we had no way to identify phase adjustments that had elevation-angle dependencies from those that did not. However, the PRM technique is not intrinsically limited to the use of one reference source for phase calibration. In fact, software within the NRAO's AIPS package (AIPS task ATMCA; Fomalont \& Kogan 2005) is specifically intended to make use of multiple reference sources with the PRM technique. This software became available only at the end of our VLBI campaign, and due to time and budgetary constraints, we were unable to use it The ability of this software to improve astrometric accuracy, relative to when a single reference source is used, has been demonstrated (Fomalont 2005).
}

detected only intermittently in single scans and in those cases only on the most sensitive baselines. Furthermore, our principal reference source, 3C 454.3, which we selected because of its close proximity on the sky to IM Peg ( 0.7 angular separation) and very high radio brightness, has a complex and evolving structure (see Paper II) that made necessary the inclusion of structure corrections in our model. The PMF technique cannot provide such corrections, so if we had relied upon the PMF technique for our astrometry, then we still would have needed to image 3C 454.3 separately and generate structure models for it. Likewise, IM Peg had a highly time-variable and sometimes complex structure (see Paper VII, Bietenholz et al. 2012, and Lebach et al. 1999), so we would have had to image this source separately, too.

Our solution to overcome the drawbacks associated with each of the PRM and PMF techniques was to merge the two approaches in a way that gave us the advantages and shed many of the disadvantages of both.

\subsection{Basic Description}

The basic approach behind the merged analysis (MA) technique is first to follow the approach of the PMF technique to obtain model corrections. One bases these corrections on data from only those radio sources-usually the reference sources-whose emissions are sufficiently bright that they can be reliably detected in a single scan on many baselines and hence are well suited for analysis with the PMF technique. For improved accuracy, structure corrections for these sources can be obtained separately and included in the analysis. The data from the sources - usually the target sources - that are not well suited for the PMF technique are left unweighted but used as "placeholders" so that model corrections corresponding to the observation times and positions of those sources are readily attainable via interpolation of the model results obtained from the data that are weighted. In effect, more accurate models for the target-source observables are obtained than with the conventional PRM technique, because one has full use of the PMF-technique tools and models that are (in general) superior to those available with the PRM technique. The corrected models from the PMF technique are then used with the PRM technique, in place of the models and corrections that would have otherwise been used in a conventional implementation of the PRM technique. Our MA technique thus combines the superior model-correction capabilities of the PMF technique with the superior sensitivity of the PRM technique.

\subsection{Implementation}

Here, we provide details about the way that we implemented the MA technique to analyze the IM Peg data obtained for $G P-B$.

\subsubsection{Observations}

Our observation strategy, as well as a list of the VLBI antennas we used, is provided in Paper II. We repeat some relevant points about our observations here.

We had 35 sessions of VLBI observations of IM Peg between 1997 January and 2005 July. We made all of these observations over a continuum near $8.4 \mathrm{GHz}(\lambda=3.6 \mathrm{~cm})$. Typically we used data from 12 to 14 VLBI antennas located around the world. Most of the antennas we used are within the United States.

We sequenced our observations through either three or four sources in a repeating 5.5 to 7 minute cycle. The three sources we observed in all 35 sessions were: our target source, 


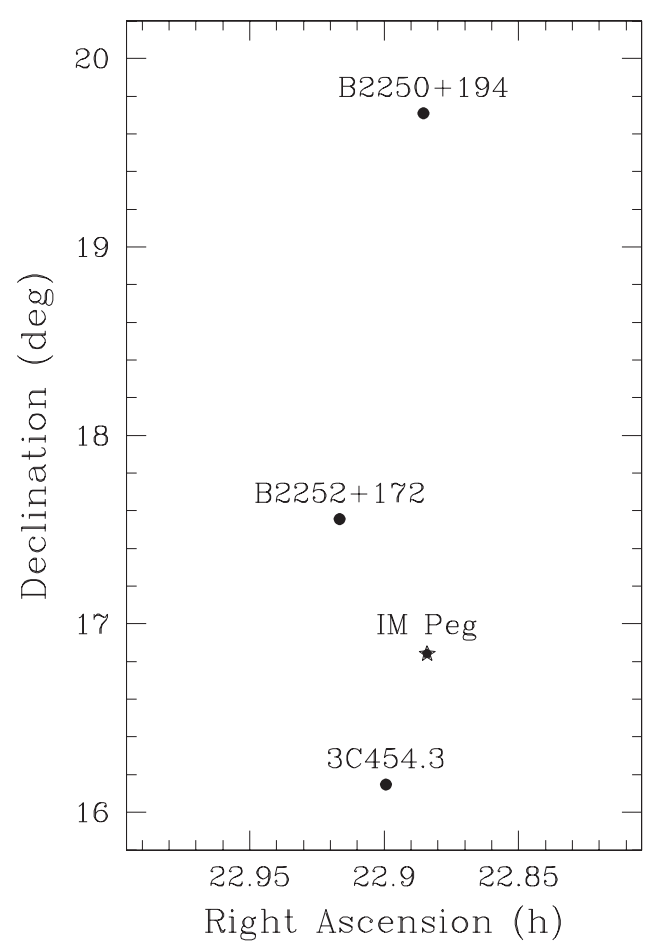

Figure 1. Positions (J2000) on the sky of the four radio sources used for $G P-B$ astrometry. The east-west and north-south directions on the plot are shown to the same scale.

IM Peg; our principal extragalactic reference source, 3C 454.3 (B2251+158); and a secondary extragalactic reference source, B2250+194. We added the fourth source, B2252+172, to our cycle for the final 12 sessions. We treated this source as a second target source and used it to place bounds on the sizes of apparent changes in the positions of our reference sources due to structural evolution (i.e., changes in the brightness distributions of the reference sources). We also used it to test our different astrometric analysis techniques. We expected B2252+172 (unlike IM Peg) to be a stationary (extragalactic) object on the sky, so any estimated motions of the source would be a measure of the experimental errors inherent in our astrometric technique. Figure 1 shows the relative positions on the sky of the four sources we observed. Note that IM Peg is between, and approximately collinear with, the reference sources 3C 454.3 and B2250+194. We intentionally selected our reference sources with such an alignment to obtain more accurate models for IM Peg data from the interpolation of the models fit to our reference-source data. These models account approximately for the elevationangle dependencies of our measured fringe phases. We selected B2252+172 primarily on the basis of its close proximity on the sky to $3 \mathrm{C} 454.3$ and secondarily for its approximate alignment, too, between 3C 454.3 and B2250+194.

The cycle time, and the scan time on each source per cycle, were chosen with the intent that they would be: (1) long enough to obtain reliable fringe-phase measurements for both reference sources on all baselines within a single scan; (2) long enough to perhaps obtain reliable fringe-phase measurements of the guide star on at least the most sensitive baselines within a single scan, thereby allowing use of the PMF technique; and (3) short enough to determine (without ambiguity) the integral number of $2 \pi$ phase wraps between successive measurements of each of the sources. We used two reference sources rather than one primarily so that we could distinguish model errors that have elevation- angle dependence (e.g., atmospheric delays) from those that do not (e.g., station clock behavior) and thereby improve our overall astrometric accuracy (see, too, Fomalont 2005).

We also analyzed data from four sessions of VLBI observations of IM Peg made by one of us and other colleagues (Lestrade et al. 1999) between 1991 December and 1994 July in support of the Hipparcos mission (Lestrade et al. 1995). However, these four sets of observations had significant differences from the later 35 sessions of observations made specifically for $G P-B$. Perhaps most significantly, these earlier observations used only four antennas per session and a single reference source, 3C 454.3. Therefore, we did not include the results from these four earlier sessions in the comparisons of the astrometric techniques that we present in this paper.

\subsubsection{First-stage Data Reduction: Calibration and Fringe Fitting}

We obtained the amplitude and phase calibration of our data for the MA technique by following steps 1-5 outlined in Section 3 for the PRM technique. We then used AIPS task FRING to estimate the fringe phases, fringe rates, and group delays from the visibility (i.e., cross-correlation) data output from the correlator. We obtained these estimates on a scan-byscan basis. We also obtained these estimates one baseline at a time by specifying two antennas at a time with the ANTENNA parameter of FRING. ${ }^{10}$ The visibility data from which these estimates for each baseline were derived were independent, and thus we followed the common (and greatly simplifying) practice of treating the estimates for each baseline as independent.

To account for the nonpointlike brightness distribution (i.e., structure) of our reference sources, we made images of the sources using the self-calibration (or "hybrid mapping") scheme described by Walker (1999). We generated one such image for each reference source for each session of observations. We obtained structure corrections for our estimated fringe phases, fringe rates, and group delays by running AIPS task FRING twice for each baseline: once with the self-calibrated CLEAN map used as input calibration and once with the source modeled as a point source (which is the FRING default). We took the structure corrections for the observables to be the differences between the estimates with a point-source map and with the CLEAN map. An important consideration in the generation of the structure corrections is the choice within the CLEAN map of the reference position that is defined to have zero structure correction (i.e., the effective point-source position of the source). Initially, we used the brightness peak of the image of each reference source as the reference position, because the brightness peak was always clearly defined and easily identifiable. However, as we discuss in Paper III (Bartel et al. 2012; see also Paper II), further studies of the collection of images of our primary reference source 3C 454.3 revealed that a different point within the source made a substantially better choice of presumed-stationary reference position. We thus adopted this point, identified as "C1" in Papers II and III, as the reference position for our 3C 454.3 structure corrections. A set of images that show the location of "C1" within the brightness distribution of 3C 454.3 can be found in Paper III.

The results from AIPS task FRING for fringe phase, fringe rate, and group delay are estimates of residuals to the model used by the correlator. We used a customized version of

\footnotetext{
10 AIPS task BLING can also be used to estimate the observables one baseline at a time. However, technical difficulties with BLING in the earliest days of our AIPS processing of the experimental data compelled us to use FRING instead, and we then opted to continue to use FRING for all experimental data.
} 


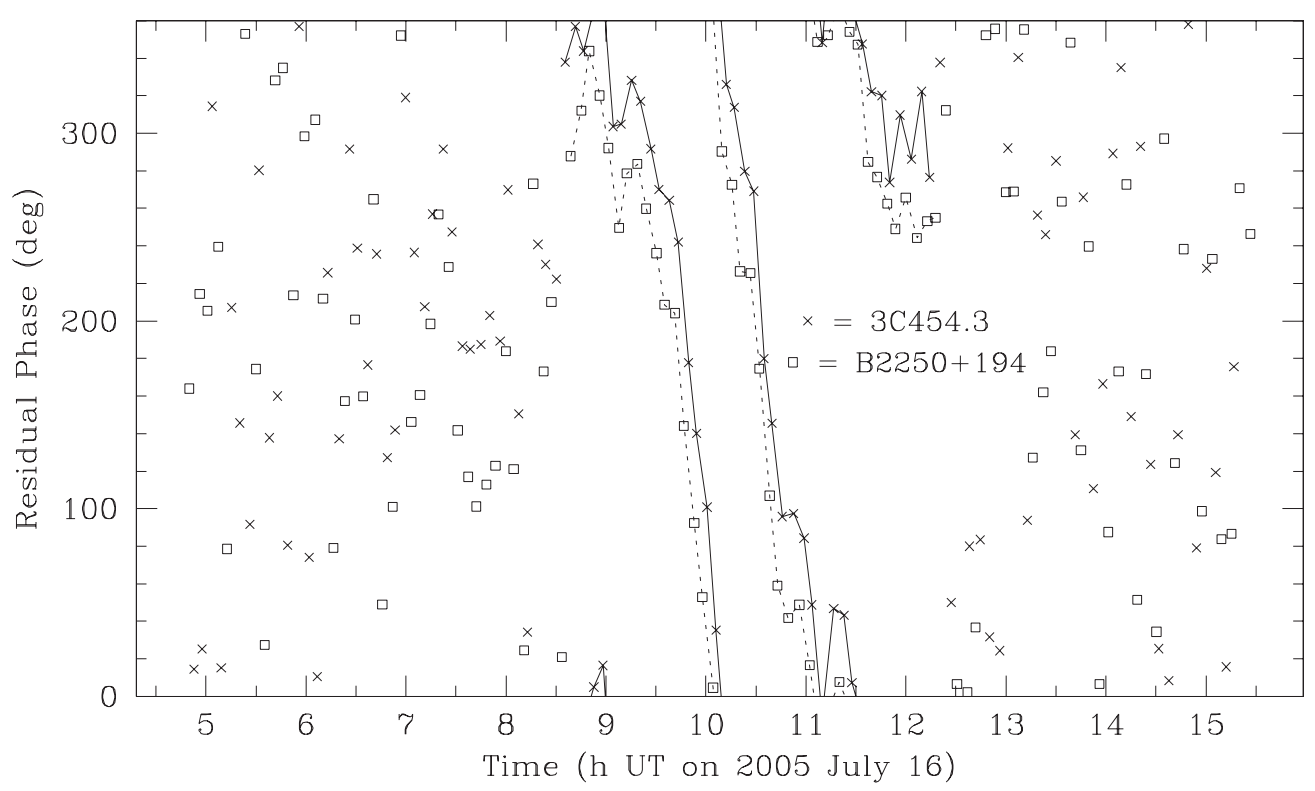

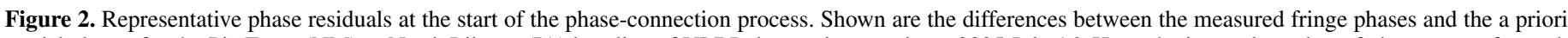

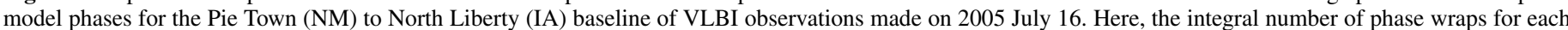

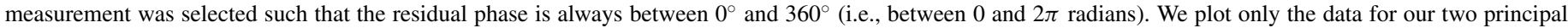

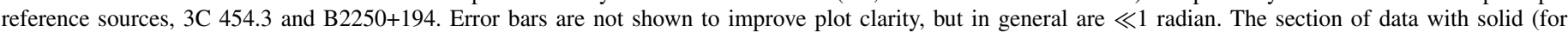

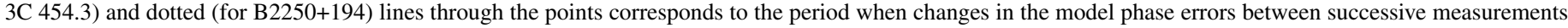

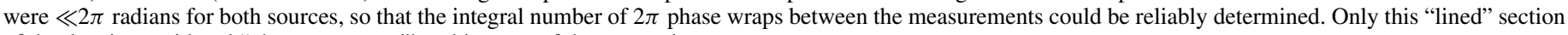
of the data is considered "phase connected" at this stage of the processing.

AIPS task CL2HF ${ }^{11}$ to add these residual estimates to the a priori model values from the correlator and place the resultant "total" observables into AIPS tables. We then "exported" these total observables from AIPS with AIPS task HF2SV. The resultant directory of files of total observables was combined with files of EOPs, surface meteorological data, antenna and source coordinates, and additional calibration data (see below) to produce databases that we used for the next stage of data reduction. We created these databases with the support of the VLBI group of the Space Geodesy Program at NASA's Goddard Space Flight Center. The theoretical observables and partial derivatives with respect to model parameters were computed with that VLBI group's CALC software (Caprette et al. 1990). We used CALC version 9.13 and the Jet Propulsion Laboratory's DE200 ephemeris (Standish 1982, 1990) for all of our a priori models.

Along with the observable data, we also exported from AIPS a collection of files of the model fringe phases and rates used by the correlator. In the final stage of our data reduction, discussed in Section 5.3.4 below, we effectively replaced these model phases and rates with improved models that we derived from the observable data that we exported.

\subsubsection{Second-stage Data Reduction: Phase Connection and Parametric Model Fitting}

The next step of our data reduction was to use the measured phases from the reference sources to improve the model estimates of the phases of the target source. In particular, we used the measured phases from our two main reference sources, $3 \mathrm{C} 454.3$ and B2250+194, to separate a priori model errors that have an elevation-angle dependence (as would be contained in $\tau_{\text {atm }}(\omega, t)$ in Equation (1)) from those that do not (as would be

\footnotetext{
11 Our customized version of this task performs the same basic functions. It includes features we required (and added ourselves) that were not available in the earliest versions of CL2HF
}

contained in $\tau_{\text {inst }}(\omega, t)$ in Equation (1)). We also used these measured phases to identify time intervals when the phases cannot be well tracked by existing models. We unweighted the targetsource data within these intervals, which could be as short as a single scan of target-source data.

"Phase connection" refers to the determination of the correct integral number of $2 \pi$ phase wraps between successive measurements of fringe phase for a particular baseline and source (see Figure 2). Phase connection is possible in part because of our very accurate a priori models and in part because of the very stable time reference (hydrogen-maser frequency standard) used at each VLBI antenna. Our basic approach to phase connection was to start with the baselines and time segments of 3C 454.3 and B2250+194 data for which our a priori models were sufficiently accurate that the number of $2 \pi$ phase wraps between successive fringe-phase measurements was immediately evident (see Figure 3). We then used this subset of phase-connected data to estimate adjustments to the parameters of our a priori model. The resulting improvement in our model enabled us to phase connect more data, and so the process proceeded in an iterative manner until we could no longer reliably phase connect any additional data. For observations above $10^{\circ}$ elevation, we reliably connected, in total, $83 \%$ of the 3C 454.3 and B2250+194 phases that we evaluated in this data-reduction stage.

The terms in Equation (1) that can be associated with difficulties in the phase-connection process are those for which the model errors can result in relatively large ( $\gtrsim 1$ radian) residual phase fluctuations in a random pattern over successive reference-source scans, i.e., in just a few minutes of time. Our a priori values for parameters such as site coordinates and EOPs were sufficiently accurate ${ }^{12}$ that they could not cause such rapid residual phase fluctuations. In fact, nearly all such phase

\footnotetext{
12 Our a priori values for antenna coordinates came primarily from the ITRF2000 solution (Altamimi et al. 2002) provided by the International Earth Rotation Service (IERS). Our a priori values for the EOPs also all came from the IERS.
} 


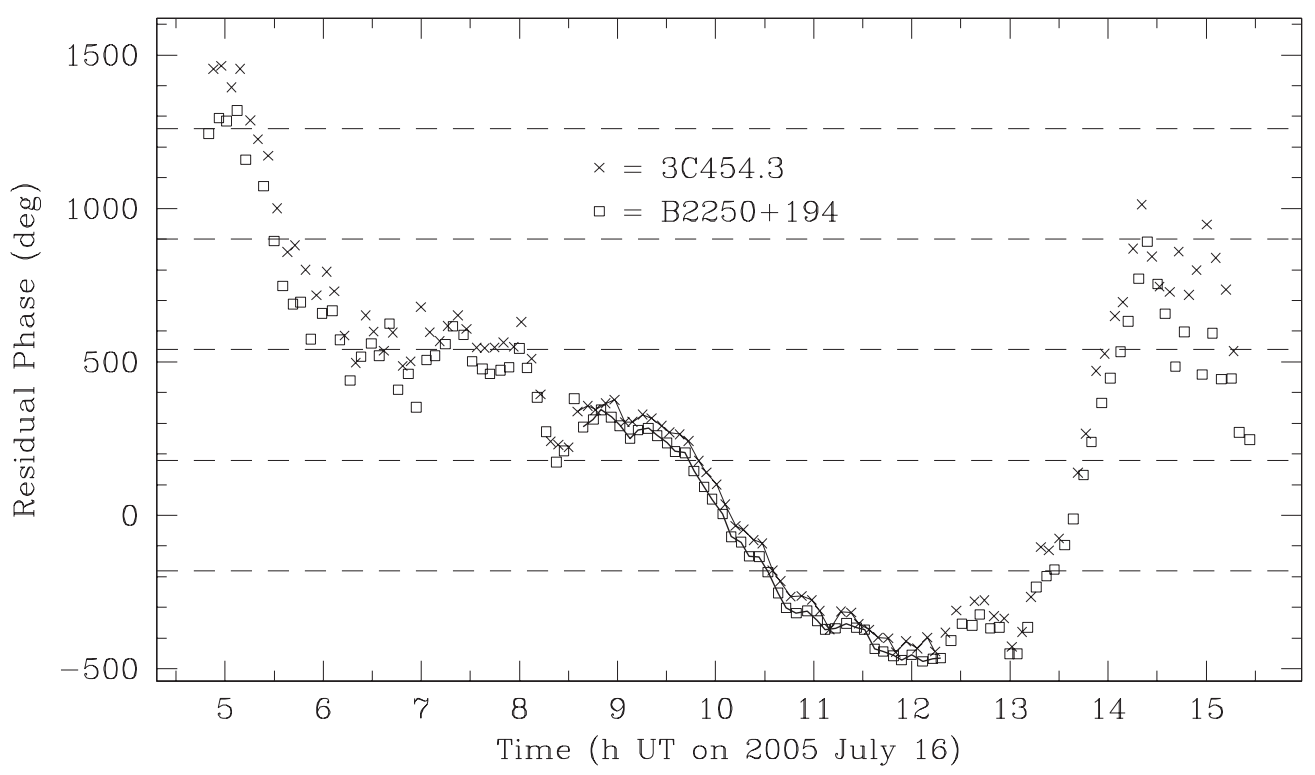

Figure 3. Same residual phases as shown in Figure 2 for the Pie Town (NM) to North Liberty (IA) baseline after the integer number of $360^{\circ}$ (or $2 \pi$ radian) phase wraps were adjusted to remove the obvious $2 \pi$ radian phase jumps. The successive dashed horizontal lines are separated by $2 \pi$ radians. The data are considered to be reliably phase-connected when the changes in the residual phases between successive measurements are $\ll 2 \pi$ radians. Only the data points with solid lines through them (one line for the 3C 454.3 data and one line for the B2250+194 data) are assumed to be reliably phase connected at this stage. The phase-connected residuals still span a range $>2 \pi$ radians; this result is due to inaccuracies in the preceding model fit that a subsequent model fit would, in general, remove. The newly phase-connected data are used in a subsequent model fit to estimate corrections to the previous model and thereby facilitate the phase connection of additional data. This process can be iterated until no more $2 \pi$ phase wraps yield residual phase changes $\ll 2 \pi$ radians between successive measurements.

fluctuations could be attributed to changes either in instrumental delays, $\tau_{\text {inst }}(\omega, t)$, which include possible "clock jumps" in the frequency standards at the sites, or, most commonly, in propagation delays through the atmosphere, $\tau_{\mathrm{atm}}(\omega, t)$. The data that we could not reliably phase-connect tended to involve sites with large wet atmospheric delays (the St. Croix VLBA site was generally the most problematic for phase connection) or be from observations just above the $10^{\circ}$ elevation threshold.

We used surface meteorological data (barometric pressure, temperature, and humidity or dew point) along with the equations from Saastamoinen (1972) to obtain a priori estimates of $\tau_{\text {zen_dry }}(t)$ and $\tau_{\text {zen_wet }}(t)$. For the mapping functions $m_{\text {dry }}(t, \epsilon)$ and $m_{\text {wet }}(t, \epsilon)$ we used the formulae provided by Niell (1996, $2000)$. Our values for $\tau_{\text {ion }}(\omega, t, \hat{\mathbf{s}})$ came from the United States Air Force's Parameterized Ionosphere Model (PIM; Daniell et al. 1995), which we adapted for use with VLBI observables (Campbell 1999). ${ }^{13}$

In fitting our data to a model, we used a Kalman-filter estimator ("SOLVK"; Herring et al. 1990) that allowed us to model atmospheric delays at zenith and instrumental drifts as Gauss-Markov stochastic processes. Our VLBI data do not provide sufficient information to simultaneously estimate adjustments to the separate terms in Equation (3); thus, we adjusted only $\tau_{\text {zen_wet }}(t)$, the term we assumed to have the least accurate a priori model. Figure 4 shows the adjustments to $\tau_{\text {zen_wet }}(t)$ that we obtained with our Kalman-filter estimator for the data corresponding to Figures 2 and 3.

\footnotetext{
13 One can obtain more accurate (we found) values for $\tau_{\text {ion }}(\omega, t, \hat{\mathbf{s}})$ via the use of AIPS task TECOR and publicly available maps of total electron content (TEC) derived from GPS data (Walker \& Chatterjee 1999). However, we found no useful TEC maps for our VLBI observations prior to 1998 September. To avoid introducing possible systematic errors into our astrometric results from the use of different ionosphere models during different periods of observations, we opted to use PIM for all of our VLBI experiments. Ionosphere model (i.e., PIM) errors contribute insignificantly to the total standard errors in the astrometric results for $G P-B$ that we present in Paper V (Ratner et al. 2012).
}

Through the use of data from two reference sources, 3C 454.3 and B2250+194, we were able to distinguish model adjustments to $\tau_{\mathrm{atm}}(\omega, t)$, which has elevation-angle dependencies, from model adjustments to other terms, e.g., $\tau_{\text {inst }}(\omega, t)$, which do not. Large phase jumps between successive reference-source scans were generally attributed to atmospheric fluctuations. Since the size of atmospheric fluctuations roughly scaled with the signal path length through the atmosphere, phase connection was generally easiest when the observed sources were at high elevation angles. Thus, we generally started our phaseconnection process with data obtained during the middle of an observing session, when the sources were at their highest elevation angles at antennas near the middle of the array. We then worked our way "outward" toward the early and late scans of the session.

When we could not confidently determine the integral number of $2 \pi$ phase wraps between consecutive reference-source scans, we unweighted the target-source data between those scans. We also inserted a "break" marker at the point of the undetermined phase jump; the Kalman-filter estimator takes account of such breaks in its model fit.

Figure 5 illustrates target-source residuals to the phase models derived from the reference-source data. The integral numbers of $2 \pi$ phase wraps between successive scans are reliably determined for all weighted data.

\subsubsection{Final-stage Data Reduction: Use of Improved Model in Phase-referenced Maps}

Once we had phase-delay and fringe-rate models based on parameter fits to the reference-source data, we imported those models into AIPS to use as the basis for our phase-referenced maps of the target source, either IM Peg or B2252+172. First we created files of fringe-phase and fringe-rate differences between the models derived from the reference-source data and the models (from the correlator) originally used in AIPS. Then we 


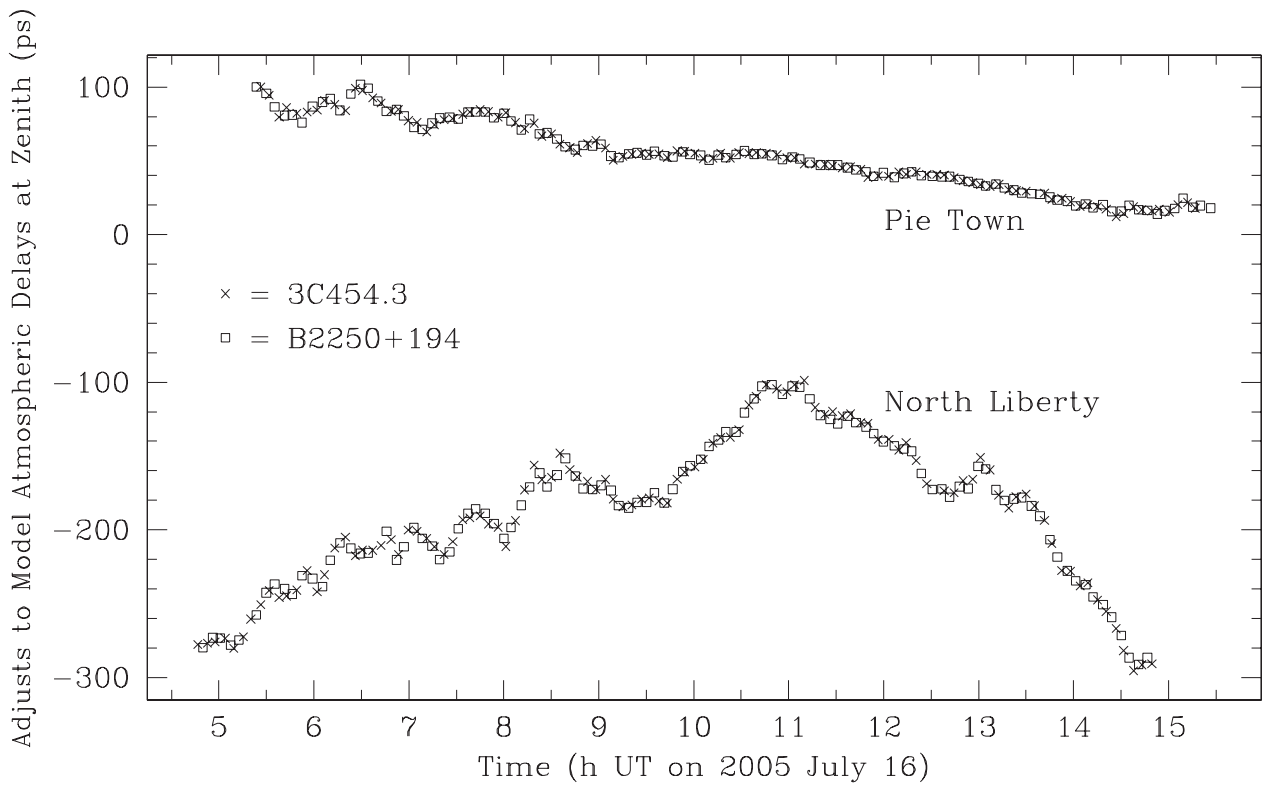

Figure 4. Adjustments to model "wet" atmospheric delays at zenith $\left(\tau_{\text {zen_wet }}\right)$ estimated from the data with our Kalman filter for two VLBI sites, Pie Town (NM) and North Liberty (IA), for our 2005 July 16 observations. For scale, a $2 \pi$ radian wrap in fringe phase corresponds to 119 ps of delay along the line of sight from each antenna to each observed source. Although the adjustments are modeled as wet delays at zenith, they include contributions from model errors in the ionosphere, too, since those errors also have elevation-angle dependence.

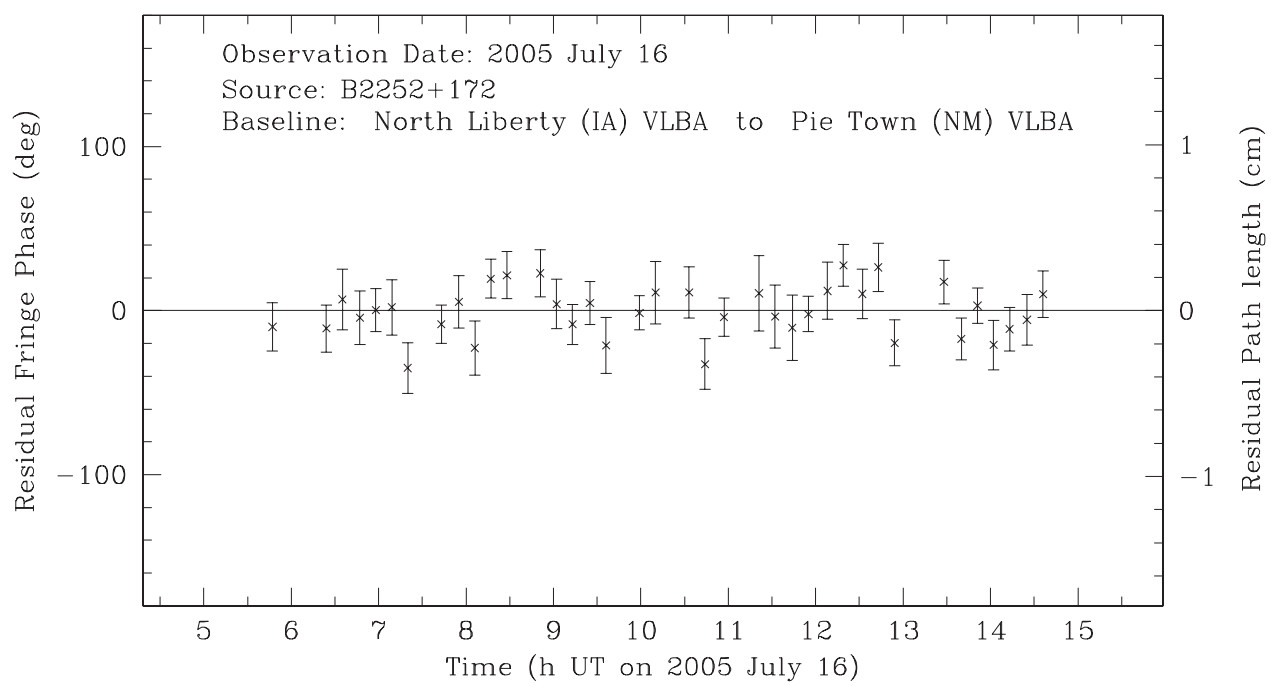

Figure 5. Sample phase residuals for source B2252+172. The phase model was derived from our reference-source (3C 454.3 and B2250+194) data with our Kalmanfilter estimator. The residuals shown are for the North Liberty (IA)-Pie Town (NM) baseline and are from our 2005 July 16 observations. The short gaps occasionally seen between adjacent data points are due (in general) either to insufficient signal strength from B2252+172 to detect the source in a single scan of data or to difficulties in determining the integral number of $2 \pi$ phase wraps between adjacent reference-source data. The number of $2 \pi$ phase wraps chosen for each point was the number that minimized the magnitude of the residual. These magnitudes, and the differences in phase residuals between adjacent scans, are all $\ll 2 \pi$ radians $\left(\ll 360^{\circ}\right)$, which demonstrates that the data are properly phase connected. We used a relatively conservative estimate for the error of each phase measurement, so the scatter of the points relative to the size of the error bars appears somewhat small.

used AIPS task TBIN to create an AIPS "solution" (SN) table with these differences. We could then treat the change from the original models used in AIPS to the improved models derived from our reference-source data as a standard AIPS calibration step. We used AIPS task CLCAL to incorporate these model adjustments. We then made phase-referenced maps with AIPS task IMAGR following standard procedures (e.g., Beasley \& Conway 1995). We obtained positions for our target sources from these IMAGR maps as described in Paper VI (Ransom et al. 2012b).

\section{COMPARISON OF RESULTS}

We used two figures of merit to compare the results of our MA technique with the more conventional PRM and PMF techniques: the quality, as described below, of the B2252+172 and IM Peg images produced (MA and PRM techniques only) and the level of consistency of the astrometric results for each of these two sources.

\subsection{Comparison of Images}

Figure 6 shows a representative map of B2252+172 produced via the conventional PRM technique alongside the corresponding map generated via our MA technique. Table 1 lists the brightness-peak flux density, the dynamic range (i.e., the ratio between the flux density of the brightness peak and the flux density of the image noise floor), and the ratio of the peak-brightness amplitude $\left(A_{\mathrm{pk}+}\right)$ to the amplitude of the most negative peak 

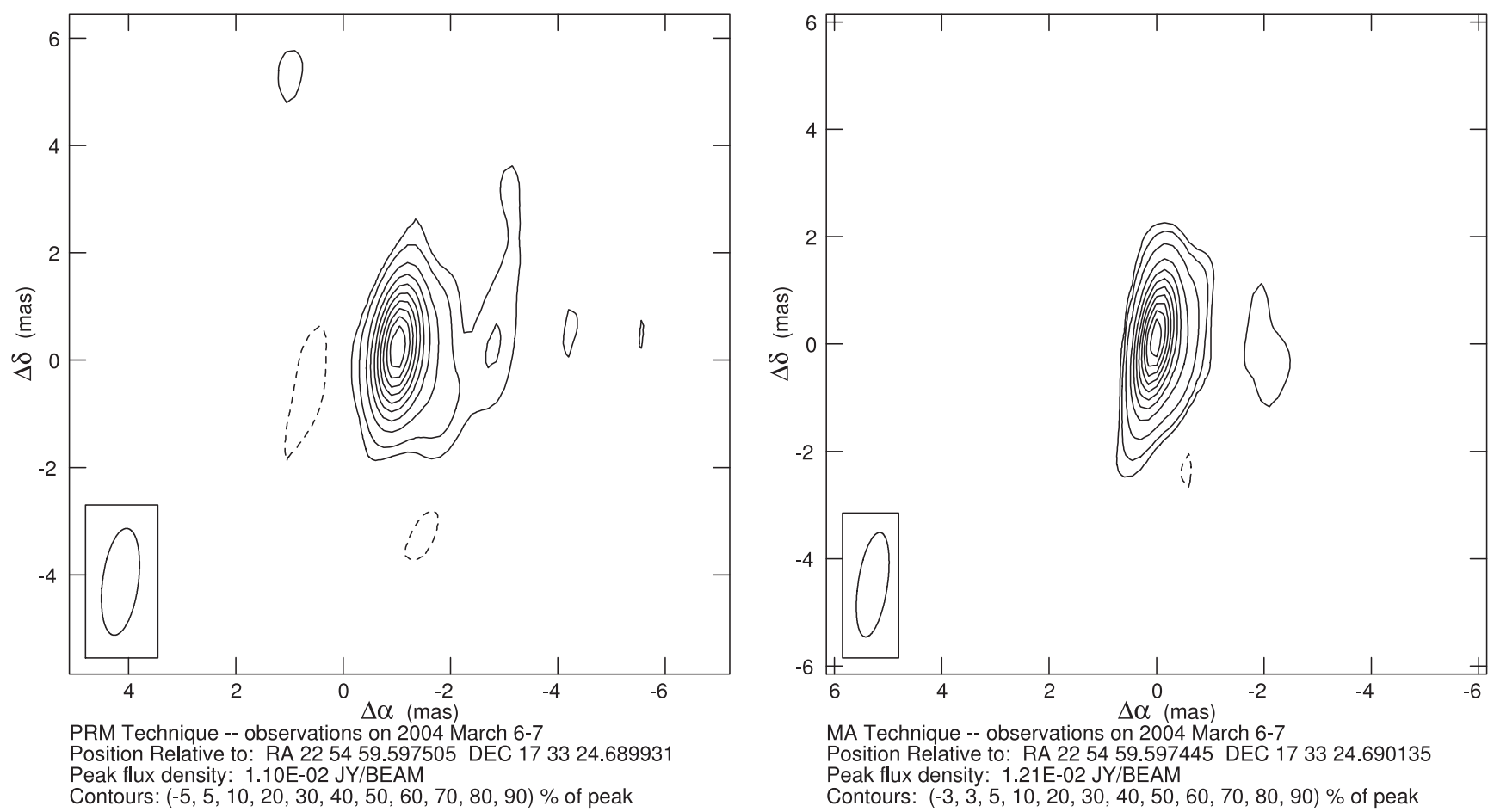

Figure 6. Representative images of B2252+172 created in AIPS with the PRM technique (left) and the MA technique (right). The data for the images are from our observations on 2004 March 6-7. Note that the two images have different coordinate origins. For each image, the size and orientation of the restoring beam is shown in the box in the lower left corner. The image created with the MA technique is visibly cleaner even with a lower minimum brightness contour. On average over all of our sessions of observations, the MA images had 1.9 times the dynamic range of the PRM images.

Table 1

Characteristics of B2252+172 Images Obtained from the PRM and MA Techniques

\begin{tabular}{|c|c|c|c|c|c|c|c|c|c|c|c|c|}
\hline \multirow{3}{*}{$\begin{array}{l}\text { Session } \\
\text { Start Date }\end{array}$} & \multicolumn{6}{|c|}{ PRM Technique } & \multicolumn{6}{|c|}{ MA Technique } \\
\hline & \multicolumn{3}{|c|}{$\begin{array}{c}\text { With } 10^{\circ} \\
\text { Elevation-angle Cutoff } \\
\text { Dyn. }\end{array}$} & \multicolumn{3}{|c|}{$\begin{array}{c}\text { With } 30^{\circ} \\
\text { Elevation-angle Cutoff } \\
\text { Dyn. }\end{array}$} & \multicolumn{3}{|c|}{$\begin{array}{c}\text { With } 10^{\circ} \\
\text { Elevation-angle Cutoff } \\
\text { Dyn. }\end{array}$} & \multicolumn{3}{|c|}{$\begin{array}{c}\text { With } 30^{\circ} \\
\text { Elevation-angle Cutoff } \\
\text { Dyn. }\end{array}$} \\
\hline & $A_{\mathrm{pk}+}{ }^{\mathrm{a}}$ & Range $^{\mathrm{b}}$ & $\frac{A_{\mathrm{pk}+\mathrm{c}} \mathrm{c}}{A_{\mathrm{pk}-}}$ & $A_{\mathrm{pk}+}$ & Range & $\frac{A_{\mathrm{pk}+}}{A_{\mathrm{pk}-}}$ & $A_{\mathrm{pk}+}$ & Range & $\frac{A_{\mathrm{pk}+}}{A_{\mathrm{pk}-}}$ & $A_{\mathrm{pk}+}$ & Range & $\frac{A_{\mathrm{pk}+}}{A_{\mathrm{pk}-}}$ \\
\hline 2002 Nov 20 & 12.2 & 64 & 9.7 & 14.2 & 68 & 13. & 15.2 & 165 & 37. & 15.5 & 170 & 31. \\
\hline 2003 Jan 26 & 11.3 & 53 & 5.6 & 14.1 & 70 & 8.3 & 17.0 & 140 & 29. & 17.9 & 149 & 38. \\
\hline 2003 May 18 & 12.5 & 62 & 7.8 & 16.6 & 60 & 8.7 & 19.1 & 187 & 47. & 19.8 & 173 & 44. \\
\hline 2003 Sep 8 & 11.7 & 64 & 13. & 13.7 & 72 & 16. & 15.4 & 141 & 34. & 16.0 & 132 & 34. \\
\hline 2003 Dec 5 & 11.0 & 51 & 7.2 & 14.3 & 61 & 9.9 & 15.4 & 106 & 16. & 16.0 & 97 & 20. \\
\hline 2004 Mar 6 & 8.9 & 56 & 8.8 & 11.0 & 76 & 12. & 12.1 & 124 & 30. & 12.4 & 110 & 25. \\
\hline 2004 May 18 & 6.5 & 37 & 7.3 & 8.8 & 48 & 8.8 & 10.0 & 77 & 18. & 10.5 & 68 & 18. \\
\hline 2004 Jun 26 & 7.0 & 54 & 11. & 9.0 & 70 & 14. & 9.8 & 109 & 25 & 10.1 & 103 & 22. \\
\hline 2004 Dec 11 & 9.0 & 58 & 8.9 & 12.0 & 94 & 14. & 12.2 & 128 & 34. & 12.8 & 116 & 30. \\
\hline 2005 Jan 15 & 9.7 & 87 & 12. & 11.0 & 103 & 12. & 11.3 & 175 & 39. & 11.6 & 149 & 38. \\
\hline 2005 May 28 & 11.7 & 48 & 10.0 & 15.5 & 69 & 15 & 16.3 & 125 & 36. & 16.5 & 114 & 33. \\
\hline $2005 \mathrm{Jul} 16$ & 12.5 & 64 & 11. & 15.4 & 80 & 14. & 16.0 & 127 & 27. & 16.5 & 131 & 28. \\
\hline MEAN $^{d}$ & 10.3 & 58.1 & 9.3 & 13.0 & 72.6 & 12.0 & 14.2 & 133.6 & 31.0 & 14.6 & 125.9 & 30.0 \\
\hline
\end{tabular}

Notes.

${ }^{\text {a }}$ Brightness-peak amplitude in mJy beam ${ }^{-1}$.

${ }^{\mathrm{b}}$ Dynamic range (see the text).

${ }^{\mathrm{c}}$ Ratio of the brightness-peak amplitude $\left(A_{\mathrm{pk}+}\right)$ to the amplitude of the most negative peak $\left(A_{\mathrm{pk}-}\right)$.

${ }^{\mathrm{d}}$ Unweighted mean over all sessions.

$\left(A_{\mathrm{pk}-}\right)$ that we obtained with the PRM and MA techniques for each of our 12 sessions of observations of this source. If the extent of the brightness distribution of a source is comparable to, or larger than, the size of the restoring beam of its image, then the measured peak brightness and (hence) the dynamic range are functions of the size of the restoring beam. The size (and orientation) of the restoring beam can in turn depend upon which data were weighted (among other factors) when the image was produced. Since we employed different data-selection criteria with the PRM and MA techniques and hence did not necessarily use identical data with the two techniques, we consider the $A_{\mathrm{pk}+} / A_{\mathrm{pk}-}$ ratio to be the most meaningful measure of image quality among the three Table 1 metrics.

For both the PRM and MA techniques, we show these metrics for images produced after we applied either a $10^{\circ}$ or a $30^{\circ}$ elevation-angle cutoff to the data (i.e., after we unweighted all 

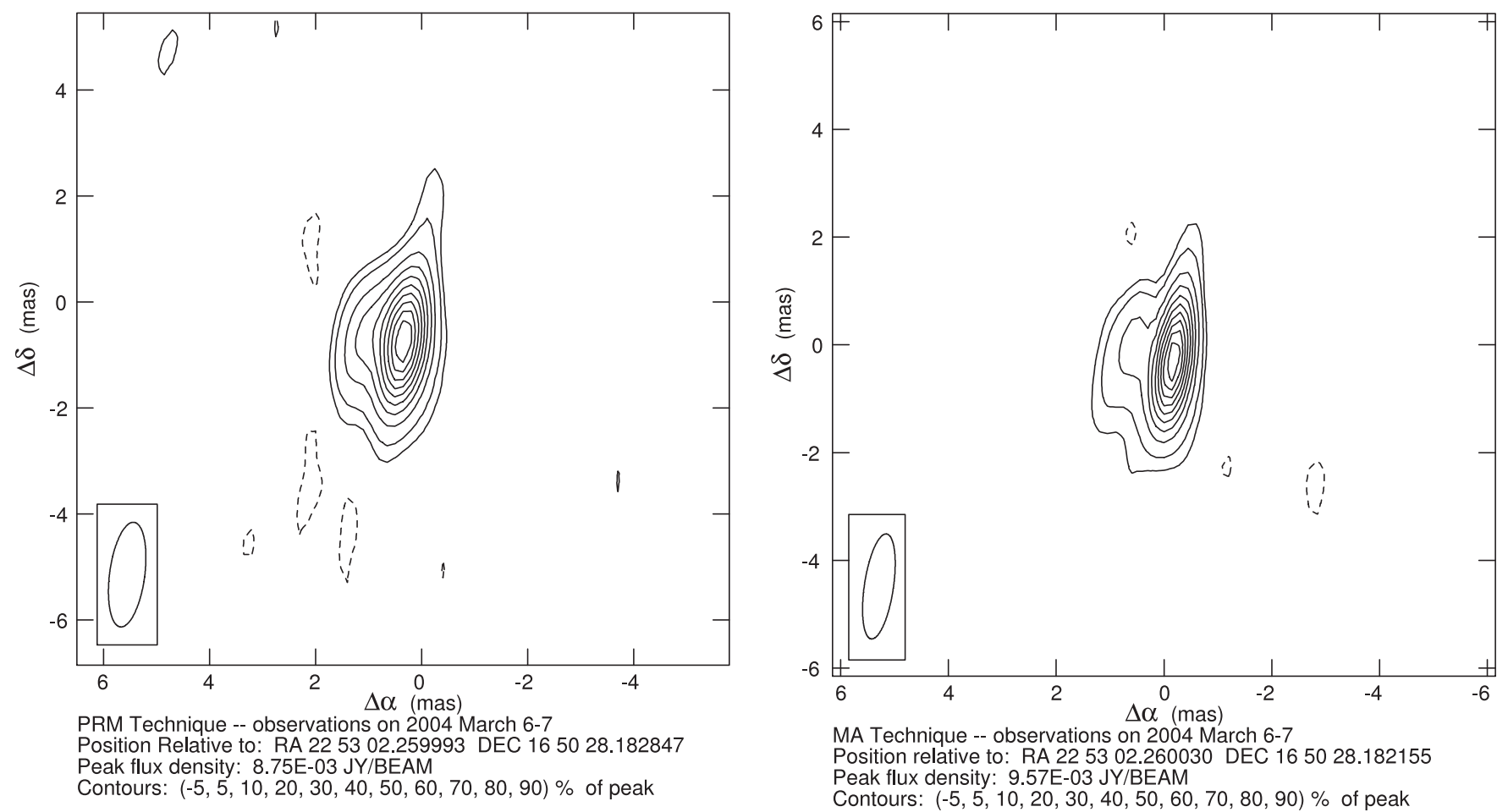

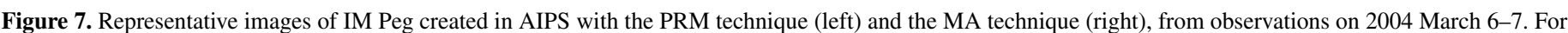

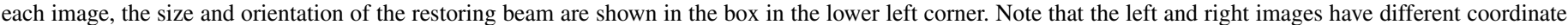
origins.

data from each site whenever the observed source was below the specified elevation angle). Since the major source of phasecalibration error with the PRM technique is often poor modeling of the atmosphere, and since (as mentioned earlier) atmospheric model errors tend to get larger at low elevation angles, the removal of low-elevation-angle data can potentially improve the quality of both the image and the astrometry obtained with the PRM technique. (Note that, for the PRM technique, the images when a $30^{\circ}$ elevation-angle cutoff is used nearly always have higher dynamic ranges and $A_{\mathrm{pk}+} / A_{\mathrm{pk}-}$ ratios than the corresponding images when a $10^{\circ}$ elevation-angle cutoff is used.) For the MA technique, only those time spans with reliably phase-connected data are weighted, so we might expect a degradation of image quality (due to the reduction of weighted data) when a higher elevation-angle cutoff is imposed. The results found for our images are mostly consistent with this expectation, although Table 1 shows that for $\mathrm{B} 2252+172$, the higher elevation-angle cutoff did improve the $A_{\mathrm{pk}+} / A_{\mathrm{pk}-}$ ratio with the MA technique in four of the twelve sessions. In any event, even if, for the PRM technique, we select for each session the elevation-angle cutoff in Table 1 with the highest dynamic range or $A_{\mathrm{pk}+} / A_{\mathrm{pk}-}$ ratio for each session, the corresponding MA images for a $10^{\circ}$ elevation-angle cutoff have, on average, 1.9 times the dynamic range and 2.7 times the $A_{\mathrm{pk}+} / A_{\mathrm{pk}-}$ ratio of the PRM images. The MA images, when compared with the PRM images, also show significantly less variability in peak flux density for the two different elevation-angle cutoffs that are used. This superior consistency, too, suggests that the MA technique provides more accurate model calibration than the PRM technique.

Figure 7 shows representative images of IM Peg produced with both the PRM and MA techniques. Averaged across all of our observing sessions, the peak flux density we detected for IM Peg was $16 \%$ higher with the MA technique than with the PRM technique. However, the significance of this result is unclear, because we used different restoring beams for each technique. Unfortunately, the data files used to make the images of IM Peg with the PRM technique were no longer available at the time of preparation of this publication, so we could not use restoring beams with the same size and orientation for both techniques. We also did not have noisefloor or "minimum peak" information available for these PRM images. In addition, we know that IM Peg can vary in total brightness and brightness distribution (i.e., radio structure) over hour timescales (Paper VII, Bietenholz et al. 2012; Lebach et al. 1999), which further complicates comparisons between the techniques, especially since a constant flux density over the full duration of a session of observations is inherently assumed in the imaging process for both techniques. Thus, for comparison of image quality from the PRM and MA techniques, we considered the B2252+172 results a better measure than the IM Peg results.

\subsection{Comparison of Astrometric Results}

Figure 8 compares the position estimates for B2252+172 (relative to 3C 454.3) from the PRM, PMF, and MA techniques. (We used a $30^{\circ}$, rather than $10^{\circ}$, elevation-angle cutoff of data with the PRM technique, because the higher cutoff yielded better results for this technique. We used our nominal $10^{\circ}$ elevationangle cutoff of data with the PMF and MA techniques.) Table 2 shows the root-mean-square (rms) scatter about the mean position for each technique. When, as with B2252+172, the target source is commonly detected on many baselines in a single scan and structure corrections with the PMF technique are available, the MA technique shows no astrometric advantage over the PMF technique. However, our MA technique yields position estimates with only about $60 \%$ of the rms scatter of the estimates from the PRM technique, i.e., removes the equivalent 


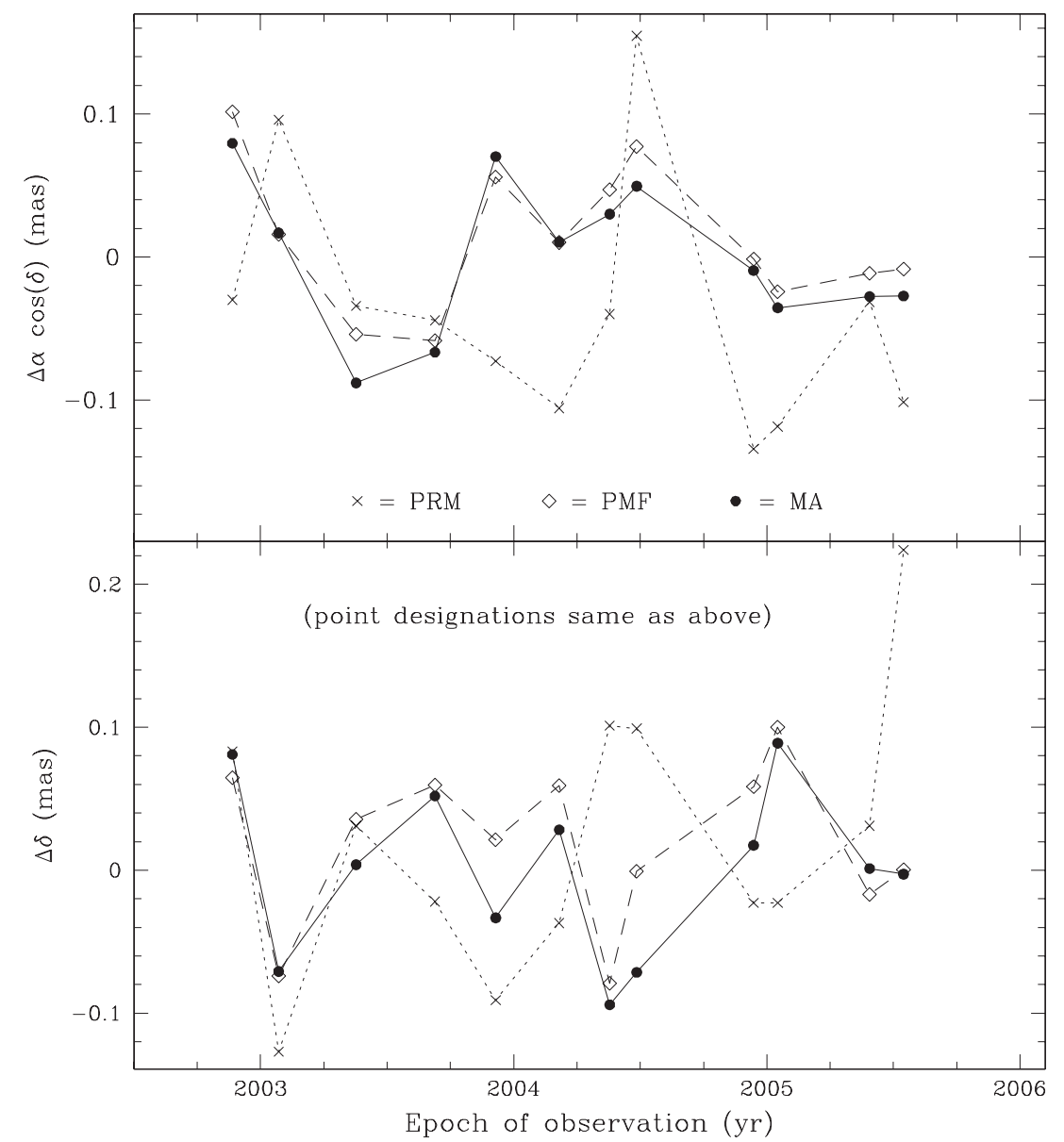

Figure 8. Position estimates for B2252+172 relative to 3C 454.3 from the PRM (diagonal crosses; connected by dotted line segments), PMF (diamonds; connected by dashed line segments), and MA (filled circles; connected by solid line segments) techniques (see the text). The zero position for each coordinate is the unweighted mean value of the 12 estimates obtained with the MA technique. For better plot clarity, the error bars for the plotted points are not shown. Since the scatter in the position estimates is due mainly to model errors (such as for the ionosphere) rather than to $\mathrm{S} / \mathrm{N}$ limits of the data, the rms scatter of the position estimates relative to the mean for each technique, as given in Table 2, is a good approximation for the errors of the corresponding points shown in this plot. Such errors ignore possible systematic effects that could have shifted the mean positions for each of the three techniques.

Table 2

Astrometric Results for B2252+172 from the PRM, PMF, and MA Techniques

\begin{tabular}{|c|c|c|c|c|c|c|}
\hline \multirow[b]{2}{*}{ Technique } & \multicolumn{2}{|c|}{$\begin{array}{c}\text { Mean Offset } \\
\text { (mas) }\end{array}$} & \multicolumn{2}{|c|}{$\begin{array}{c}\text { RMS Scatter } \\
\text { (mas) }\end{array}$} & \multicolumn{2}{|c|}{$\begin{array}{l}\begin{array}{l}\text { Standard Error } \\
\text { of Mean }^{\mathrm{c}} \text { (mas) }\end{array} \\
\end{array}$} \\
\hline & $\alpha$ & $\delta$ & $\alpha$ & $\delta$ & $\alpha$ & $\delta$ \\
\hline PRM & -0.039 & 0.021 & 0.082 & 0.092 & 0.025 & 0.028 \\
\hline PMF & 0.012 & 0.019 & 0.048 & 0.053 & 0.014 & 0.016 \\
\hline MA & 0.000 & 0.000 & 0.050 & 0.057 & 0.014 & 0.017 \\
\hline
\end{tabular}

Notes. For each technique we assumed, for each of $\alpha$ and $\delta$, the same standard error for every observing session. The rms scatter for the PRM technique is the largest; the levels of rms scatter for the PMF and MA techniques are not significantly different from each other, although the former appear to be slightly smaller.

${ }^{a}$ Defined to be zero for the MA technique in both right ascension $(\alpha)$ and declination $(\delta)$. The mean offsets for the PRM and PMF techniques are relative to the mean coordinate position from the MA technique.

${ }^{\mathrm{b}}$ Relative to the mean coordinate position for each technique.

${ }^{\mathrm{c}}$ Calculated from the rms scatter.

of a noise source of over 0.06 mas per coordinate estimate associated with the PRM technique.

The model for IM Peg's position over time must include proper motion, parallax, and orbital motions associated with the binary system. We used the preferred parameterization of Paper V (Ratner et al. 2012), one in which no proper acceleration is estimated, to obtain the results presented here.

For 7 of the 35 observing sessions of IM Peg, including the most astrometrically important final three, the radio emissions from the star were insufficiently bright to obtain reliable detections of IM Peg in single scans. Thus, we could not obtain position estimates of IM Peg with the PMF technique for any of these sessions. We also found from images obtained with the PRM and MA techniques that IM Peg had relatively complex structure during several of our observing sessions (see Paper VII), and the PMF technique by itself cannot well identify or characterize such structure. Thus, we deemed the PMF technique unsuitable for astrometric studies of IM Peg with our data.

In Figure 9 we show the post-fit residuals of the position estimates for IM Peg from the PRM and MA techniques, and in Table 3 the rms scatter of these same post-fit residuals. On average the post-fit residuals from our MA technique had about $10 \%$ less scatter in right ascension and $7 \%$ less scatter in declination than the corresponding residuals from the PRM technique. The scatter from both techniques is likely dominated by the unmodeled intrinsic motions of the stellar radio emission relative to the star's primary component (Paper VI). Hence, since we have no model that can accurately describe the motion 


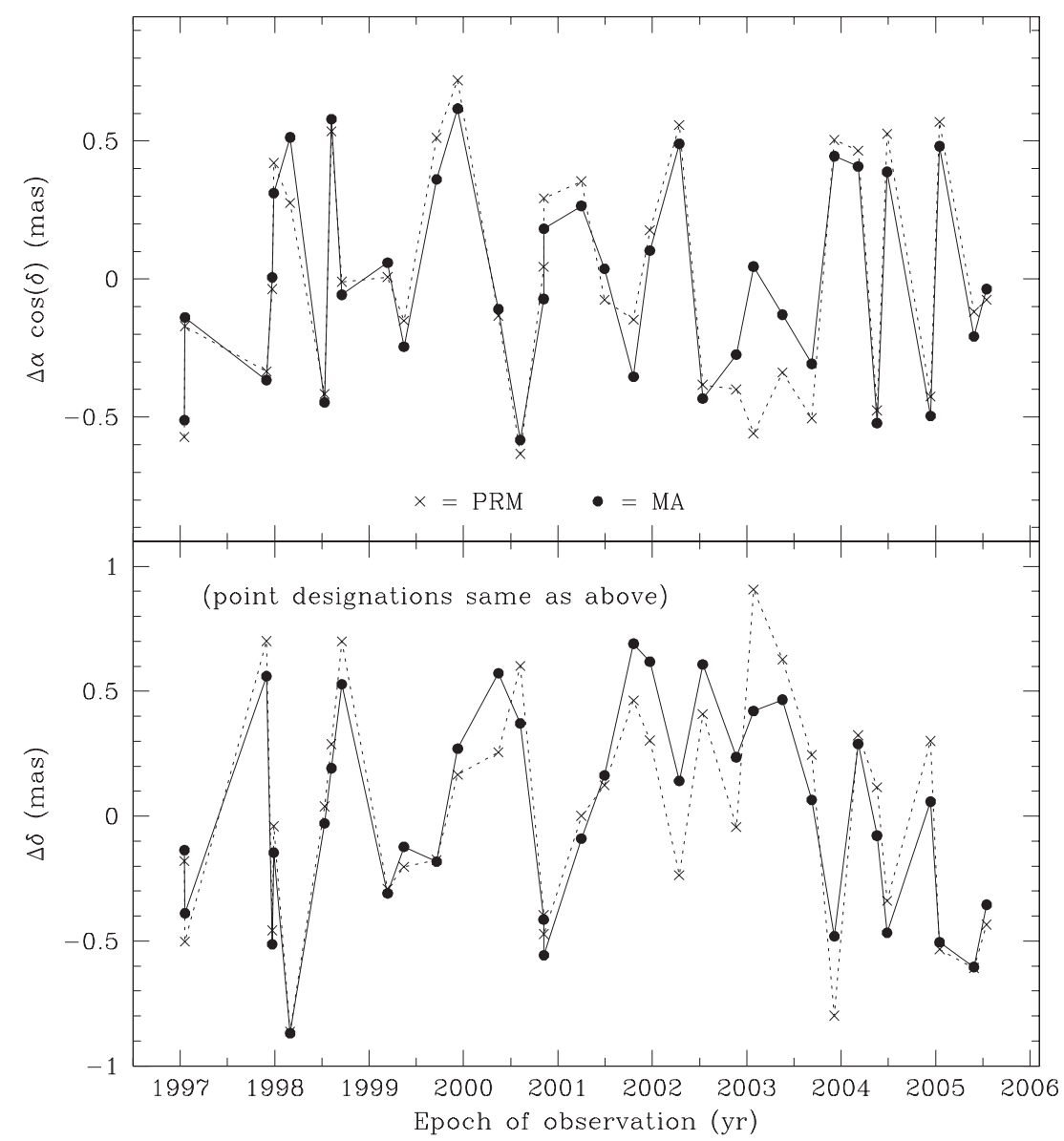

Figure 9. Post-fit residuals of the position estimates of IM Peg from the PRM (diagonal crosses; connected by dotted line segments) and MA (filled circles; connected by solid line segments) techniques. (In the model fit, we estimate the position, proper motion, parallax, and apparent binary-system orbit of the stellar radio emitting region.) We could not obtain position estimates of IM Peg with the PMF technique for 7 of our 35 observing sessions due to insufficient radio brightness of the star; hence we deemed the standard PMF technique unsuitable for astrometric studies of IM Peg with our data. For better plot clarity, the error bars for the plotted points are not shown. The scatter in the position estimates from both the PRM and MA techniques is due mainly to intrinsic motions of the radio emission of IM Peg relative to the primary component of the star rather than to errors in the position estimates of the radio emission.

Table 3

RMS Scatter of IM Peg Position Residuals Obtained from the PRM and MA Techniques

\begin{tabular}{lcc}
\hline \hline & \multicolumn{3}{c}{ RMS Scatter } \\
(mas) & \\
Technique & $\alpha$ & \\
\hline PRM & 0.40 & 0.44 \\
MA & 0.35 & 0.41 \\
\hline
\end{tabular}

Notes. The position residuals are relative to an astrometric model fit in which the radio emissions are assumed to be tied to a single stellar component of the IM Peg binary system. We believe that the rms scatter is due predominantly to intrinsic motions of the radio emissions relative to this stellar component rather than to measurement accuracy. Nevertheless, the rms scatter is roughly $10 \%$ smaller with the MA technique than with the PRM technique.

of the radio emission of IM Peg relative to its primary, we can expect at most only a minor improvement in the rms scatter of position residuals from the use of any superior astrometric technique. Indeed the MA technique yielded just such a minor improvement.

\section{CONCLUSIONS}

In comparing the astrometric results from PRM, PMF, and the MA technique introduced in this paper, we found the following.

1. The PRM technique can be used with relatively weak radio sources. It is the least labor intensive among the techniques tested, but it also provides the least astrometric accuracy. (With this technique, unlike with the PMF or MA techniques, we were unable to use multiple reference sources to better account for the elevation-angle dependence of model errors; such use could improve the astrometric accuracy attainable with this technique.)

2. The PMF technique is useful only when both reference and target sources are: (1) reliably detectable in short (i.e., few-minute) scans of data; and (2) pointlike, unless structure corrections are available from other means. Under these circumstances, the PMF technique yielded the highest astrometric accuracy among the techniques tested.

3. Our MA technique can provide astrometric accuracies nearly equal to those obtained with the PMF technique.

4. Very importantly, our MA technique can be used with sources that are too weak for use of the PMF technique, as was IM Peg during 7 of our 35 VLBI sessions. In general, any source that is sufficiently bright for use of the PRM technique is sufficiently bright for use of our MA 
technique. The MA technique can also be used with sources that have complex brightness distributions and was shown to yield images with significantly higher dynamic ranges than comparable images from the PRM technique.

Our use of the MA technique enabled us to obtain more accurate astrometric measurements of IM Peg, the guide star for the GP$B$ mission, than we could have otherwise obtained with the conventional PRM or PMF techniques.

We thank N. Nunes for developing the graphical software package we used to phase-connect our VLBI data. We thank the VLBI group of NASA's Goddard Space Flight Center, and L. Petrov in particular, for providing technical support and information. We are also grateful to the many people involved in our campaign of VLBI observations who went above and beyond the call of duty, including S. Dains, C. Garcia Miro, E. Moll, and L. Cameron. We thank C. Jacobs and O. Sovers for information about the coordinates of the three $70 \mathrm{~m}$ NASA DSN antennas used in our observations, and R. C. Walker for information about the VLA coordinates. We thank J. L. Davis and S. S. Shapiro for their information and insight on the use of our Kalman-filter estimator (SOLVK). Finally, we thank R. C. Walker, K. Desai, and E. Greisen for their support in our use of AIPS. The National Radio Astronomy Observatory is a facility of the National Science Foundation operated under cooperative agreement by Associated Universities, Inc. This research made use of NASA's Astrophysics Data System, which was conceived, developed, and continues to be operated by the Smithsonian Astrophysical Observatory at the Harvard-Smithsonian Center for Astrophysics. Our work was supported by NASA prime award NAS8-39225, Stanford University subaward PR 6750, the Smithsonian Institution, and York University.

\section{REFERENCES}

Altamimi, Z., Sillard, P., \& Boucher, C. 2002, J. Geophys. Res.(Solid Earth), 107,2

Bartel, N., Bietenholz, M. F., Lebach, D. E., et al. 2012, ApJS, 201, 3 (Paper III)

Bartel, N., Ratner, M. I., Shapiro, I. I., et al. 1985, AJ, 90, 318

Beasley, A. J., \& Conway, J. E. 1995, in ASP Conf. Ser. 82, Very Long Baseline Interferometry and the VLBA, ed. J. A. Zensus, P. J. Diamond, \& P. J. Napier (San Francisco, CA: ASP), 327

Bietenholz, M. F., Bartel, N., Lebach, D. E., et al. 2012, ApJS, 201, 7 (Paper VII)

Campbell, R. M. 1999, New Astron. Rev., 43, 617

Caprette, D. S., Ma, C., \& Ryan, J. W. 1990, NASA Technical Memorandum 100765 (Crustal Dynamics Project Data Analysis; Greenbelt, MD: NASA Goddard Space Flight Center)

Clark, T. A., Corey, B. E., Davis, J. L., Herring, T. A., \& Elgered, G. 1985, IEEE Trans. Geosci. Remote Sens., 23, 438

Counselman, C. C., III, Kent, S. M., Knight, C. A., et al. 1974, Phys. Rev. Lett., 33,1621

Daniell, R. E., Brown, L. D., Anderson, D. N., et al. 1995, Radio Sci., 30, 1499
Davis, J. L., Herring, T. A., Shapiro, I. I., Rogers, A. E. E., \& Elgered, G. 1985, Radio Sci., 20, 1593

Diamond, P. J. 1995, in ASP Conf. Ser. 82, Very Long Baseline Interferometry and the VLBA, ed. J. A. Zensus, P. J. Diamond, \& P. J. Napier (San Francisco, CA: ASP), 227

Dodson, R., Legge, D., Reynolds, J. E., \& McCulloch, P. M. 2003, ApJ, 596, 1137

Fey, A. L., Gordon, D., \& Jacobs, C. S. (ed.) 2009, IERS Technical Note No. 35, http://www.iers.org/IERS/EN/Publications/TechnicalNotes/tn35.html (Frankfurt: Verlag des Bundesamts für Kartographie und Geodäsie)

Fomalont, E. B. 2005, in ASP Conf. Ser. 340, Future Directions in High Resolution Astronomy, ed. J. Romney \& M. Reid (San Francisco, CA: ASP), 460

Fomalont, E. B., \& Kogan, L. 2005, AIPS Memo 111 (ATMCA: Phase Referencing Using More Than One Calibrator; Socorro, NM: NRAO), ftp://ftp.aoc.nrao.edu/pub/software/aips/TEXT/PUBL/AIPSMEM111.PS

Gorenstein, M. V., Shapiro, I. I., Cohen, N. L., et al. 1983, Science, 219, 54

Herring, T. A., Davis, J. L., \& Shapiro, I. I. 1990, J. Geophys. Res., 95, 12561

Herrnstein, J. R., Moran, J. M., Greenhill, L. J., et al. 1999, Nature, 400, 539

Ishihara, Y., Nakai, N., Iyomoto, N., et al. 2001, PASJ, 53, 215

Kondratko, P. T., Greenhill, L. J., \& Moran, J. M. 2004, in Proc. 222nd IAU Symp., The Interplay Among Black Holes, Stars, and ISM in Galactic Nuclei, ed. T. Storchi-Bergmann, L. C. Ho, \& H. R. Schmitt (Cambridge: Cambridge University Press), 325

Lebach, D. E., Ratner, M. I., Shapiro, I. I., et al. 1999, ApJ, 517, L43

Lestrade, J.-F., Jones, D. L., Preston, R. A., et al. 1995, A\&A, 304, 182

Lestrade, J.-F., Preston, R. A., Jones, D. L., et al. 1999, A\&A, 344, 1014

Lestrade, J.-F., Rogers, A. E. E., Whitney, A. R., et al. 1990, AJ, 99, 1663

Ma, C., Arias, E. F., Eubanks, T. M., et al. 1998, AJ, 116, 516

Mathews, P. M., \& Shapiro, I. I. 1995, Phys. Earth Planet. Inter., 92, 99

Niell, A. E. 1996, J. Geophys. Res., 101, 3227

Niell, A. E. 2000, Earth Planets Space, 52, 699

Pradel, N., Charlot, P., \& Lestrade, J.-F. 2006, A\&A, 452, 1099

Ransom, R. R., Bartel, N., Bietenholz, M. F., et al. 2012a, ApJS, 201, 2 (Paper II)

Ransom, R. R., Bartel, N., Bietenholz, M. F., et al. 2012b, ApJS, 201, 6 (Paper VI)

Ratner, M. I., Bartel, N., Bietenholz, M. F., et al. 2012, ApJS, 201, 5 (Paper V)

Rodin, A., \& Sekido, M. 2002, in Proc. 6th EVN Symp., ed. E. Ros, R. W. Porcas, A. P. Lobanov, \& J. A. Zensus (Bonn: Max-Planck-Institut für Radioastronomie), 247

Rogers, A. E. E., Hinteregger, H. F., Whitney, A. R., et al. 1974, ApJ, 193, 293

Ryan, J. W., Ma, C., \& Caprette, D. S. 1993, NASA Technical Memorandum 104572 (Data analysis 1992, Final Report of the Crustal Dynamics Project VLBI Geodetic Results, 1979-1991; Greenbelt, MD: NASA Goddard Space Flight Center)

Saastamoinen, J. 1972, in The Use of Artificial Satellites for Geodesy, ed. S. W. Henriksen, A. Mancini, \& B. H. Chovitz (Washington, DC: AGU), 247

Seidelmann, P. K. 1982, Celest. Mech., 27, 79

Seidelmann, P. K. (ed.) 1992, Explanatory Supplement to the Astronomical Almanac (Mill Valley, CA: Univ. Science Books)

Shapiro, I. I., Bartel, N., Bietenholz, M. F., et al. 2012, ApJS, 201, 1 (Paper I)

Shapiro, I. I., Wittels, J. J., Counselman, C. C., III, et al. 1979, AJ, 84, 1459

Shapiro, S. S., Davis, J. L., Lebach, D. E., \& Gregory, J. S. 2004, Phys. Rev. Lett., 92, 121101

Standish, E. M., Jr. 1982, A\&A, 114, 297

Standish, E. M., Jr. 1990, A\&A, 233, 252

Walker, R. C. 1999, in ASP Conf. Ser. 180, Synthesis Imaging in Radio Astronomy II, ed. G. B. Taylor, C. L. Carilli, \& R. A. Perley (San Francisco, CA: ASP), 433

Walker, R. C., \& Chatterjee, S. 1999, VLBA Scientific Memo 23 (Ionospheric Corrections Using GPS Based Models; Socorro, NM: NRAO), http://www.vlba.nrao.edu/memos/sci/ 